\title{
Psychological aspects of excimer laser surgery for myopia: reasons for seeking treatment and patient satisfaction
}

\author{
Charles N J McGhee, Dianne Orr, Brian Kidd, Cameron Stark, Iain G Bryce, \\ Con $\mathrm{N}$ Anastas
}

SEI Corneal Diseases and Excimer Laser Unit, Sunderland Eye Infirmary, Sunderland SR2 9HP

C N J McGhee

D Orr

I G Bryce

C N Anastas

Edith Morgan Centre, Department of Psychiatry, Torbay Hospital, Torbay TQ2

7AA

B Kidd

Department of Public Health, Ayrshire and Arran Health Board, Ayrshire KA7 4DW C Stark

University of

Sunderland, School of

Health Sciences,

Sunderland SR2 3SD

C N J McGhee

D Orr

Correspondence to:

Professor C N J McGhee, Dundee University

Department of

Ophthalmology, Ninewells

Hospital and Medical

School, Dundee DD1 9SY.

Accepted for publication 16 July 1996

\begin{abstract}
Aims-To determine the reasons patients seek excimer laser treatment for myopia and to measure levels of patient satisfaction with each phase of this relatively new treatment.

Methods-A unique 38 item visual analogue questionnaire was developed and applied to 100 consecutive patients $(n=$ 100) who had undergone excimer laser photorefractive keratectomy for myopia or myopic astigmatism at a single centre. All subjects underwent extensive ophthalmic assessment and patient education before treatment.

Results-A $90 \%$ response rate was achieved $(n=90)$. Improved unaided vision $(85.6 \%)$, freedom from spectacles (83.3\%), and difficulties with contact lens wear $(72.7 \%)$ were the most common reasons for seeking treatment. The majority of patients felt that the quality of their life had improved $(85.5 \%)$ and $82 \%$ felt that the quality of their unaided vision was as good as they had anticipated. Overall, $95.5 \%$ of patients were pleased that they had undergone this procedure. The vast majority $(98.9 \%)$ of patients felt that they fully understood the operation pretreatment but almost one quarter (23.9\%) found the actual operation distressing. Only $15.7 \%$ found postoperative pain did not respond adequately to prescribed analgesia.

Conclusions-This study demonstrates a very high level of patient satisfaction after excimer laser treatment for myopia and myopic astigmatism. It suggests that most patients seek treatment to enjoy better vision free from optical aids and that a high level of patient education preoperatively may establish realistic expectations and satisfaction with overall outcome. (Br F Ophthalmol 1996;80:874-879)
\end{abstract}

The first excimer laser treatment upon a human eye to correct refractive error was carried out by Seiler et al in $1988 .{ }^{1}$ Since then the widespread use of the excimer laser for the treatment of myopia and astigmatism has steadily increased; however, it is still a relatively new concept in eye care and consequently few studies have been carried out on patient satisfaction following excimer laser surgery. Previous results from studies carried out by telephone ${ }^{23}$ and questionnaire ${ }^{4}$ have indicated a high level of patient satisfaction $(84.6 \%$ to $92 \%) .^{2-4}$ Also, a survey of surgeons' impressions of patient satisfaction after radial keratotomy recorded a $96 \%$ level of satisfaction ${ }^{5}$ and the Prospective Evaluation of Radial Keratotomy (PERK) study results confirm that patients are generally satisfied with the long term visual results following myopic correction by radial keratotomy. ${ }^{6}$

Patient satisfaction has been measured in relation to many other forms of medical treatment and has traditionally been seen as a measure of the quality of that treatment. A proved relation exists between patient satisfaction and use of a service, ${ }^{7}$ with dissatisfied patients moving from one service provider to another. ${ }^{8}$ Satisfied patients are more likely to comply with treatment ${ }^{9}$ and studies of satisfaction can give valuable information to providers of a service regarding possible improvement in areas such as communication and mode of treatment delivery. ${ }^{10}$ Previous studies that have attempted to identify patients' rationale for undergoing PRK and levels of postoperative satisfaction have tended to use relatively crude measures. To improve the delivery of patient oriented ophthalmic care in general, and excimer laser provision in particular, more sensitive indices of patient satisfaction are required. Carefully constructed satisfaction studies are not merely for the purpose of reassuring the practitioner that a specified number of patients are 'satisfied' but should rather form the basis for critical analysis and improvement of service.

In neurology and mammography clinics, patients have been shown to report a high level of overall satisfaction; however, when asked specific questions regarding topics such as communication, support from staff, or the amount of pain experienced during a procedure the same patients may also express numerous complaints and reservations. ${ }^{.1}$ Therefore, while measures of overall satisfaction may be comforting to a service, they provide little information regarding the specific subcomponents of satisfaction, the measurement of which might provide a service with much better guidance for improvement. After reviewing the literature, Hall and Dornan, in 1988, identified 12 dimensions of patient satisfaction..$^{10}$ In their review they illustrated that those items which tended to be scored highly appeared most often in satisfac- 
tion studies; it therefore follows that studies based on the existing literature will be unlikely to ask about items which tend to show patient dissatisfaction. Fitzpatrick has summarised the advantages and disadvantages associated with the types of questionnaire available, ${ }^{12}$ noting that most scales are developed by the workers who use them, frequently with little attempt to analyse their face or content validity. This lack of questionnaire standardisation therefore makes it difficult to compare satisfaction between different services, even within the same specialty. The selection of different subject groups may further complicate analysis. The limited data published to date, regarding patient satisfaction following excimer laser PRK, demonstrate the difficulty in overcoming these problems.

In order to address some of the problems inherent in the assessment of patient satisfaction following excimer laser PRK, the authors developed a unique questionnaire which they have applied to a large cohort of consecutive patients undergoing excimer laser treatment for myopic refractive disorders.

\section{Subjects}

The first 100 consecutive patients (male: female ratio 51:49) to undergo excimer laser photorefractive keratectomy (PRK) or photoastigmatic refractive keratectomy (PARK) at the Sunderland Eye Infirmary, Corneal Diseases and Excimer Laser Unit, between the period May 1993 and November 1993 were identified. These subjects (mean age 39.4 (SD 11.2 ) years, range $20-68$ years) were a subset of a major prospective study $(n=454)$ analysing the effectiveness of excimer laser treatment for myopic refractive errors. All patients were assessed and treated by two surgeons (CNJM and IGB). All patients received a six page patient information guide outlining the risks, benefits, and predicted outcome of excimer laser treatment before their first assessment. In addition, an average of 90 minutes was allotted for the assessment of each patient preoperatively, including extended discussion of likely outcome and risks, with one or both surgeons. Finally, a two page, 10 paragraph informed consent form was discussed with, and signed by, the subjects before treatment.

All patients were treated on a Visx 20/20 excimer laser (Visx Inc, Sunnyvale, CA, USA) with both surgeons present. Preoperative myopic spherical equivalent ranged from $-0.87 \mathrm{D}$

11 Do you think it was useful to be reviewed the day after the operation?

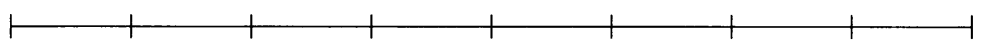

Not useful at all

Very useful

Figure 1 Sample question showing linear analogue scale anchored by adjectival descriptors. to $-20.00 \mathrm{D}$ (mean -5.84 (SD 3.47) D). Postoperative assessments were performed at days 1,3 , and 7 , with monthly follow up reviews until month 6 post-treatment. Thereafter, reviews were at 3 monthly intervals until 2 years post-treatment. A standard postoperative drug regimen was prescribed in all cases which consisted of diclofenac (Voltarol, Cibavision) 1 drop 4 to 6 hourly for 48 hours (if required for pain); chloramphenicol $0.5 \%$ (Chloromycetin, ParkeDavis) four times daily for 7 days; fluorometholone $0.1 \%$ (FML Liquifilm, Allergan UK Ltd) four times daily for 90 days; and Co-proxamol (dextropropoxyphene $30 \mathrm{mg}$ with paracetamol $325 \mathrm{mg}$ ) two tablets 4 to 6 hourly for 48 hours.

\section{Method}

In conjunction with psychiatrists and public health colleagues a unique questionnaire was developed. Since satisfaction is not a unidimensional construct-that is, patients may be 'satisfied' with the overall outcome, but dissatisfied with components of care or subcomponents of outcome-for example, the necessity to wear spectacles occasionally, an extensive questionnaire was constructed. A total of 38 questions were devised which covered six main topics related to patient care, satisfaction, and patients' reasons for undergoing this form of surgery. The topics analysed included: (1) reasons for undergoing excimer laser treatment (eight questions); (2) preoperative clinical assessment (seven questions); (3) excimer laser operative procedure (five questions); (4) immediate postoperative care (five questions); (5) long term postoperative care (six questions); (6) final outcome of treatment (seven questions).

The questions from each topic group were mixed randomly within the compiled questionnaire to avoid preferential response bias within these subgroups. Rather than limit patient response options in a 'yes/no' format, which forces patients into 'black and white' choices, a visual analogue scale, anchored at each end by adjectival description, was chosen for each question (Fig 1)

A questionnaire was posted to each patient together with a stamped addressed envelope, a pen, and a covering letter explaining the reason for this research. The patient sample was compiled by a research assistant (DO) who was not part of the clinical team. The covering letter reassured patients of the proposed anonymity of the study and that the data would be analysed by the research assistant and not the clinicians involved with the provision of service. Patients were therefore invited to complete the questionnaire as truthfully and critically as possible and were informed that all data would be held anonymously. Each questionnaire was identified by a unique number which was later correlated with data held, anonymously, but with an identical number identifier on a computer database. The results from all of the returned questionnaires were transferred to a computer database. One month after the first letters were sent out a gentle reminder was posted and more replies 
were returned. Ninety replies (90\%) were received from 100 questionnaires forwarded. The overall responses to each question were calculated from the database with patient responses on each analogue scale being subdivided into four equal categories for the purposes of analysis: (1) very positive response, (2) positive response, (3) negative response, (4) very negative response. No 'neutral' response group was derived for analysis and in very uncommon examples where a patient's response fell exactly upon one of the division lines (breaking the analogue scale into four categories), the response was classified in the lower category-for example, if the patient's mark fell on the division between 'very positive response' and 'positive response', the comment was classified as 'positive response'.

\section{Results}

DEMOGRAPHICS AND UNAIDED VISUAL ACUITY Of 100 consecutive patients who had undergone excimer laser PRK/PARK and who were invited to participate in this study, 90 subjects $(90 \%)$ completed and returned the questionnaire (mean age 40.1 (SD 11.1) years, range $21-61$ years, $M: F$ ratio $43: 47$ ). At the time of questioning 86 of all 100 patients involved in the study had unaided vision of $6 / 12$ or better. (Three of those whose unaided vision was less than $6 / 12$ also had preoperative best corrected visual acuity less than 6/12.) Among responders $87.7 \%$ had unaided vision of $6 / 12$ or better $(88.8 \%$ when patients whose best corrected preoperative vision was less than $6 / 12$ are excluded), whereas among nonresponders $70.0 \%$ had unaided vision of $6 / 12$ or better $(87.5 \%$ when patients whose best corrected preoperative vision was less than $6 / 12$ are excluded). Therefore, no significant differences in sex, age, or unaided visual outcome were observed between responders and non-responders in this study and the responders can therefore be considered a large representative sample $(90 \%)$ of 100 consecutive patients.

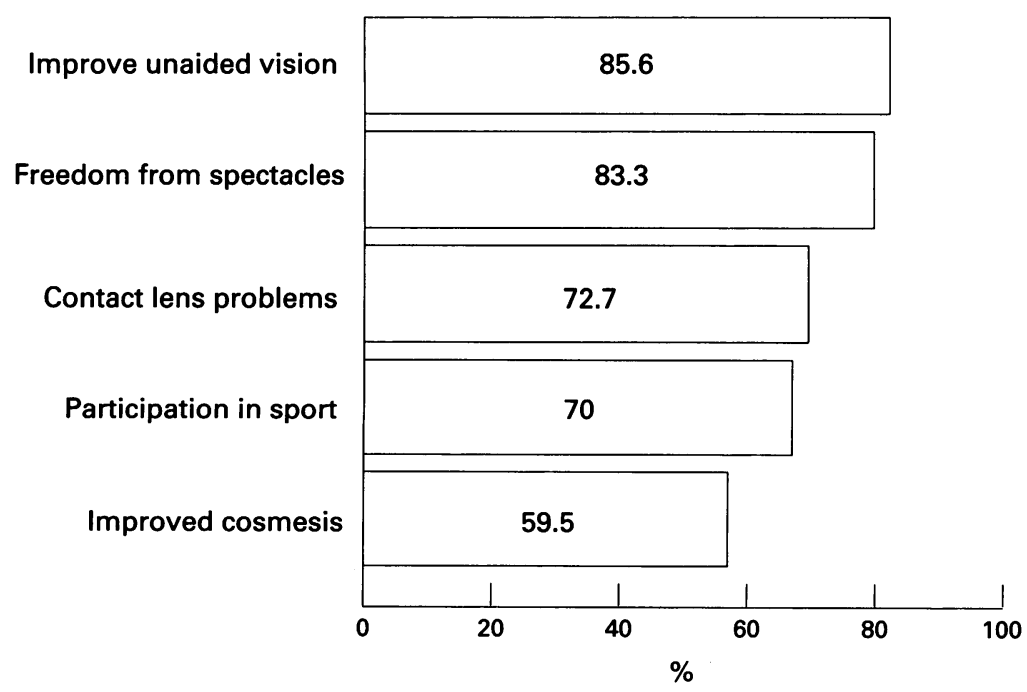

Figure 2 Summary of patients' responses with regard to reasons for undergoing excimer laser correction of myopia and myopic astigmatism $(n=90)$.
REASONS FOR UNDERGOING EXCIMER LASER TREATMENT

The most important reason stated for undergoing excimer laser treatment was to improve unaided vision $(85.6 \%$, very positive $78.9 \%)$ or to be free from spectacles $(83.3 \%$, very positive $70 \%$ ), whereas $72.7 \%$ (very positive $59.1 \%$ ) reported difficulties with contact lens wear as being very important. Improved unaided vision enabling subjects to participate in sport $(70.0 \%$, very positive $52.2 \%)$ and improved cosmetic appearance $(59.5 \%$, very positive $30.3 \%$ ) were also considered important (Fig 2).

PREOPERATIVE INFORMATION AND ASSESSMENT Verbal preoperative information was thought to be sufficient by $94.5 \%$ (very positive $88.9 \%$ ) of patients and $95.5 \%$ (very positive $74.2 \%$ ) of respondents also expressed the opinion that written information provided before treatment was also sufficiently extensive. Most patients ( $95.6 \%$, very positive $94.5 \%$ ) believed that their questions were answered adequately. All patients $(100 \%$, very positive $95.5 \%)$ reported that before treatment they were assessed thoroughly. All patients ( $100 \%$, very positive $96.7 \%$ ) noted that any further information they might require was readily available. The procedure was fully understood by $98.9 \%$ (very positive $92.2 \%$ ) and many felt that talking to patients who had already undergone the procedure made them feel more comfortable about the surgery $(84.1 \%$, very positive $51.2 \%)$.

\section{OPERATIVE PROCEDURE}

The vast majority of patients noted that their operation was performed professionally $(98.9 \%$, very positive $97.7 \%)$ and that they had no difficulty following the surgeon's instructions $(94.4 \%$, very positive $93.3 \%$ ) during the operation, while $94.4 \%$ (very positive $83.2 \%$ ) regarded the operation procedure was as they had expected. The wait before the operation was considered too long by $31.5 \%$ (very positive $20.2 \%$ ) of patients and $23.9 \%$ (very positive $8 \%$ ) found the actual operation distressing.

IMMEDIATE POSTOPERATIVE CARE

A high percentage $(98.9 \%$, very positive 92.2\%) of patients reported that medical staff were readily available to allay their fears and $\mathbf{9 7 . 8 \%}$ (very positive $\mathbf{8 8 . 8 \%}$ ) indicated that staff explained adequately what to do about pain management after the operation. Some $\mathbf{9 4 . 4 \%}$ (very positive $91.1 \%$ ) felt it was useful to be reviewed the day immediately after the operation and $94.5 \%$ (very positive $90.0 \%$ ) felt medications were explained adequately. Although $84.3 \%$ (very positive $71.9 \%$ ) believed they received sufficient medications to ease the degree of postoperative pain to a tolerable level, $15.7 \%$ did not.

\section{LONG TERM POSTOPERATIVE CARE}

Subjects generally agreed that they were reviewed sufficiently frequently postoperatively (93.3\%, very positive $72.2 \%$ ), and the majority $(85.4 \%$, very positive $69.7 \%$ ) were happy with 
the speed of visual improvement. When questioned upon their ability to function well after only one eye had been treated $80.7 \%$ (very positive $59.1 \%$ ) were able and $19.3 \%$ were not able to function well. Only $10.2 \%$ (very positive $3.4 \%$ ) of patients expressed difficulty in applying postoperative topical medication. When referred to the question 'Did you discuss the operation adequately with other patients who had undergone the treatment', $65.2 \%$ (very positive $39.3 \%$ ) stated they had done so and $77.7 \%$ (very positive $45.9 \%$ ) stated that talking to patients after the operation made them feel more satisfied with their final visual result.

\section{LONG TERM OUTCOME}

A summary of patient responses regarding long term outcome of excimer laser treatment is contained in Table 1. Overall, $95.5 \%$ (very positive $84.3 \%$ ) of patients were happy, or very happy, that they had undergone this operation (Fig 3). There were four subjects who felt unhappy $(n=2)$ or very unhappy $(n=2)$ with the final outcome of the procedure. All four had a preoperative myopic spherical equivalent of $-5.0 \mathrm{D}$ or greater. Three of this group had a final unaided vision of $6 / 36$ or poorer, one had lost one Snellen line of best corrected acuity, and another had lost two lines. The fourth subject, with preoperative myopia of $-8.0 \mathrm{D}$, enjoyed unaided acuity of $6 / 9$ with no detracting visual symptoms. Despite having been advised that a final (predicted) unaided visual acuity of $6 / 18$ to $6 / 12$ would be a successful outcome at this level of myopia, this latter patient was disappointed that her vision was not better than $6 / 9$ unaided ( $6 / 5$ corrected).

Regression analysis demonstrated no correlation between preoperative myopia, postoperative myopia, or failure to achieve $6 / 12$ unaided acuity and expressed levels of satisfaction. Only weak correlations were identified between these three indices and other components of the questionnaire. A weak correlation was identified between preoperative myopia greater than $-6.00 \mathrm{D}$ and greater reliance on spectacles postoperatively $\left(r^{2}=0.15,95 \%\right.$ confidence interval (CI) $0.10<R<0.63$ ). Those subjects with unaided visual acuity less than $6 / 12$ postoperatively demonstrated a weak correlation with the preoperative desire to have treatment to be 'free from spectacles' $\left(r^{2}=\right.$ $0.13,95 \% \mathrm{CI}-0.6<R-0.05)$. While losing 2 lines of best spectacle corrected Snellen acuity (BSCVA) was weakly correlated with currently wearing a spectacle correction $\left(r^{2}=0.13,95 \%\right.$ CI; $0.05<R<0.60$ ), it was also weakly correlated with: satisfaction with speed of improvement of vision $\left(r^{2}=0.12,95 \% \mathrm{CI} ; 0.05<R\right.$

Table 1 Summary of patients'positive and very positive (in parentheses) responses with regard to questions on long term outcome of excimer laser treatment of myopia and myopic astigmatism $(n=90)$

Do you feel your quality of life has improved?

Have you achieved the goals for which you underwent the operation?

$85.5 \%(50 \%)$

Is your unaided eyesight as good as you anticipated?

Is your reliance on spectacles or contact lenses as anticipated?

$86.5 \%(67.4 \%)$

$82 \% \quad(70.8 \%)$

$71.9 \%(63.4 \%)$

$25.3 \%(19.5 \%)$

Are you currently wearing spectacles or contact lenses?

$95.5 \%(84.3 \%)$

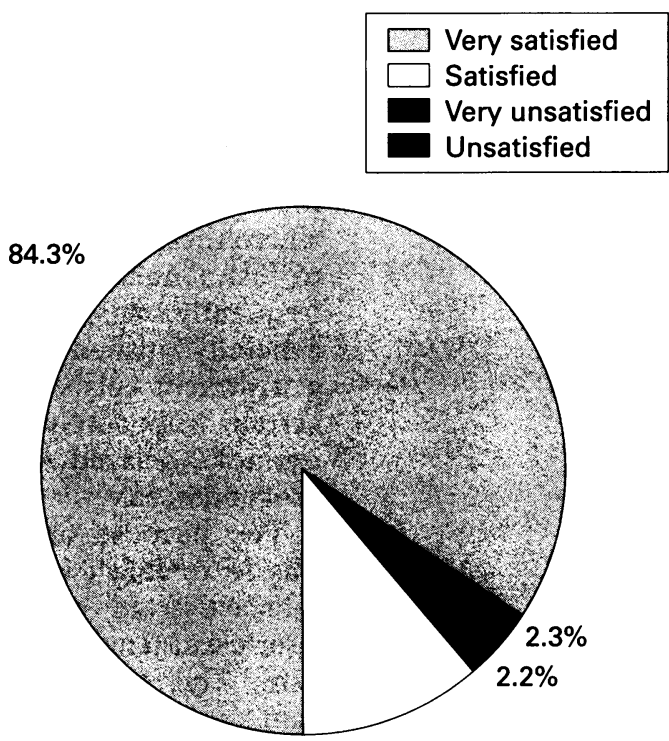

$11.2 \%$

Figure 3 Patients' responses with regard to satisfaction with overall outcome of excimer laser treatment of their myopia or myopic astigmatism $(n=90)$.

$<0.59$ ) and achievement of goals for which the procedure was undertaken $\left(r^{2}=0.18,95 \% \mathrm{CI}\right.$; $-0.65<R<-0.13$ )

\section{Discussion}

A number of studies have examined the area of satisfaction in patients receiving excimer laser PRK. When Heitzmann et al used patients' acceptance of treatment on their second eye as a crude measure of overall satisfaction with PRK for high myopia, ${ }^{14}$ it was shown that none of the 18 patients studied refused treatment. Kahle et al attempted to measure changes in quality of life and degree of satisfaction with myopic correction 1 year after PRK in 26 patients ${ }^{4}$; however, the quality of life measures were basic and were based on a choice of only three options-better, no change, or worse. Using this format the authors demonstrated an improvement in quality of life for $75 \%$ of patients, with $21 \%$ reporting no change and $4 \%$ getting worse. While $84 \%$ of patients were satisfied with their myopic correction, $16 \%$ were indifferent or dissatisfied. Gimbel et al reported results from 52 patients following bilateral PRK in which overall ratings of vision and refractive results were recorded, showing that $88.1 \%$ felt their vision to be 'good to excellent' while $11.9 \%$ felt it was only 'fair'. Again, using an unsophisticated 'overall satisfaction' measurement, $90.5 \%$ reported that they were satisfied or very satisfied with their refractive result while $9.5 \%$ were dissatisfied. ${ }^{3}$ Fichte and Bell measured similar outcomes to those listed above but used different scales. ${ }^{2}$ Unfortunately, they also failed to break the measures of satisfaction down into specific components of care, thus limiting the amount of useful information obtainable. They found $92 \%$ to be 'satisfied' overall, with $93 \%$ intending to have their second eye treated and $89 \%$ noting an improvement in their work, home, or social life. Significantly, $96 \%$ of patients stated that they would recommend excimer laser PRK to a friend. 
Unfortunately, when general questions about patient satisfaction are posed-for example, 'were you happy with the outcome/ service overall', then around $80 \%$ of respondents always answer in the affirmative. ${ }^{12}$ In order to identify areas of dissatisfaction, or areas where there is room for improvement, one has to ask much more specific questions. It frequently transpires that although patients are generally satisfied with the eventual outcome of a treatment episode, they are not happy with certain subcomponents of the service-for example, delayed recovery, discomfort of the procedure, etc. ${ }^{1213}$

The purpose of this questionnaire survey was to establish in detail the level of patient satisfaction in patients undergoing excimer laser treatment for myopia or myopic astigmatism. This also provided the opportunity to gain knowledge of other important aspects relevant to patient satisfaction, including delineation of areas of difficulty in the provision of an excimer laser service and identification of reasons for patients undergoing treatment. Since one of the objects of the study was to develop a tool which could be used in a busy clinic situation to provide meaningful feedback, the study was conducted from the centre providing the service. While administration of such a study by a third party is likely to minimise positive bias in respondents, by utilising a non-clinical research member of staff and emphasising the anonymity of the study, we hope to have minimised potential bias created by the providers of care posing the questions, yet created a reproducible local system to assess satisfaction.

The topics of the questions comprised six main groups, attempting to cover the whole treatment from preoperative assessment to the final visual outcome. Overall the level of patient satisfaction is high $(95.5 \%)$ and equals the highest levels noted in previous studies with quoted reports of patient satisfaction ranging from $84.6 \%$ to $96.0 \% .^{3-5}$ However, it is noteworthy that in these studies ${ }^{2}$ this information was gained via the telephone or in person, techniques which may well have slanted the patients' replies towards a more favourable result. Kraff reported, based on information on surgeons' subjective impressions of patient satisfaction in refractive surgery, that $96 \%$ of patients were very satisfied. ${ }^{5}$ Notably, three of four dissatisfied patients in the present study had unaided vision of $6 / 36$ or poorer, while one attained $6 / 9$ unaided vision. These results must be considered in the context of the higher preoperative myopia in this subgroup and the fact that the remaining seven respondents in this study who achieved less than 6/12 unaided acuity, were either satisfied or very satisfied with their long term outcome. Although it might be reasonably concluded that a less good unaided visual acuity might be more likely to result in dissatisfied patients, preoperative education and counselling to set realistic outcomes, and the patient's underlying (occasionally unrealistic) expectations are also major components in determining subject's final assessment of the success of the procedure.
Indeed, in the present study, regression analysis failed to show any significant correlation between a poor refractive outcome, unaided visual acuity poorer than $6 / 12$, and any of the satisfaction outcome measures. Perhaps surprisingly, those subjects losing 2 lines or more of BSCVA actually demonstrated a weakly positive correlation with positive responses to achieving the goals for which they had undergone the procedure and satisfaction with the speed of visual improvement. These data confirm the complexity of the relation between patient education, expectation, service delivery, operative outcome, and patient satisfaction. Satisfaction is not unidimensional and related to outcome solely in terms of residual refraction and unaided vision, and highly motivated and informed patients may be very satisfied with outcome and delivery of service, despite an apparently poor ophthalmic result in terms of loss of BSCVA.

Interestingly, the results of this study show that the most important stated reason for undergoing excimer laser treatment is to improve unaided vision (85.6\%) which is in keeping with a report by Kahle in which 'to improve general vision' was the highest ranked motivation. ${ }^{4}$ However, in contrast with Kahle's findings that 'looking better' was only of minor importance, $59.5 \%$ of our patients actually indicated that cosmetic appearance was an important reason for undergoing treatment and of these positive responders, $33 \%$ were male and $67 \%$ female. Perhaps surprisingly, the expense of spectacles and contact lenses was rated as an important reason for seeking excimer laser treatment by only $26.7 \%$ of respondents in the present study. However, it must be noted that many of the subjects in the present study were older and in financially secure positions (mean age 39.4 years) and that the annual cost of contact lenses or spectacles represented only $10-15 \%$ of the cost of bilateral laser treatment.

Questions concerning preoperative assessment have not previously been reported so comparisons are not available. Preoperative assessment is an extremely important part of excimer laser treatment and it is essential that patients are fully informed and their expectations are genuinely realistic with regard to the outcome of the operation. Perhaps, because of the investment of a substantial amount of time, within this unit, in addressing patients' understanding of this procedure preoperatively, a very high percentage of patients felt they were given sufficient verbal information (94.5\%) and written information (95.5\%). During assessment patients were encouraged to talk to one another and to patients who had already undergone PRK. This helped $84.1 \%$ to feel more comfortable about the treatment. Notably, this high level of patient information, and formulation of a realistic viewpoint with regard to post-treatment outcome, may well be responsible, at least in part, for the stated overall patient satisfaction with final outcome.

The information gleaned from the questions regarding the operation itself confirms that intensive preoperative patient education pays 
dividends in the perioperative period with $94.4 \%$ indicating the procedure was as expected, $98.9 \%$ expressing that the operation was performed professionally, and only $5.6 \%$ finding the surgeon's instructions difficult to follow. However, operations of any kind can make patients anxious and a long wait (1-3 hours) can only increase this anxiety such that $31.5 \%$ felt that this wait was too long and $23.9 \%$ felt the operation was distressing. These two factors may be linked; hence, a reduction in wait before treatment may reduce anxiety and lower the total number of patients feeling distressed. Immediate postoperative results indicated that despite possible inconvenience and postoperative discomfort, patients do like to be seen by medical staff on the first day after the operation $(94.4 \%)$, since this enables any fears to be allayed and problems can be discussed. Significantly, despite oral and topical analgesia, a small group of patients $(15.7 \%)$ did not feel that they received sufficient pain relief which highlights an area where further therapeutic refinements are still required.

Excimer laser PRK is a recent development in refractive surgery and in many areas is only available on an investigational basis. As such, the numbers studied are relatively small and research has tended to focus on objective clinical outcomes such as visual acuity and refractive errors, or symptoms such as the degree of 'halo' or 'glare'. The studies of satisfaction to date seem to have been secondary to the technical concerns of the surgeon and have tended to use only measures of overall satisfaction, failing to identify the components of satisfaction which may enable improvement of service delivery and ultimately outcome of this treatment.

Presented in part at the Second Annual United Kingdom International Ophthalmic Excimer Laser Congress, February 1995, Harrogate, UK.

1 Seiler T, Bende T, Wollensak J, Trokel S. Excimer laser keratectomy for correction of astigmatism. Am $\mathcal{f}$ Ophthal mol 1988;105;117-24.

2 Fichte CM, Bell AM. Ongoing results of excimer laser photorefractive keratectomy for myopia: subjective patient impressions. F Cataract Refract Surg 1994;20 (Suppl):268-70.

3 Gimbel HV, Van Westenbrugge JA, Johnston WH, Willerscheidt AB, Sun $R$, Ferensowicz $M$. Visual, refractive and patient satisfaction results following bilateral photorefractive keratectomy for myopia. Refract Corneal Surg 1993;9 (Suppl):s5-s11.

4 Kahle G, Seiler T, Wollensak J. Report on psychosocial findings and satisfaction among patients 1 year after excimer ings and satisfaction among patients 1 year after excimer laser photorefrac

5 Kraff MC, Sanders DR, Karcher D, Raanan M, Deluca M, Neumann G. Changing practice patterns in refractive surgery: results of a survey of the American Society of Cataract and Refractive Surgery. $\mathcal{F}$ Cataract Refract Surg 1994;20:172-8.

6 Bourque LB, Cosand BB, Drews C, Waring GO, Lynn M, Cartwright C. Reported satisfaction, fluctuation of vision and glare among patients one year after surgery in the Prospective Evaluation of Radial Keratotomy (PERK) study. Arch Ophthalmol 1986;104:356-63.

7 Roghmann K, Zastowney TR. Satisfaction with medical care: its measurement and relation to utilisation. Medical Care 1979;17:461-77.

8 Weiss B, Senf J. Patient satisfaction survey instrument for use in health maintenance organisations. Medical Care 1990;28:434-45.

9 Kincey J, Bradshaw P, Ley P. Patients' satisfaction and reported acceptance of advice in general practice. $\mathcal{f}$ Roy Coll Gen Pract 1975;25:558-76.

10 Hall JA, Dornan MC. What patients like about their medical care and how often they are asked: a meta-analysis of cal care and how often they are asked: a meta-analysis

11 Fitzpatrick R, Hopkins. Problem in the conceptual framework of patient satisfaction search: an empirical exploration. Soc Sci Med 1983;27:935-9.

12 Fitzpatrick R. Surveys of patient satisfaction: I-Important general considerations. BMF 1991;302:87-9.

13 Fitzpatrick R. Surveys of patient satisfaction: II-Designing a questionnaire and conducting a survey. $B M \Im$ 1991;302: 1129-32.

14 Heitzmann J, Binder PS, Kassar BS, Nordan LT. The correction of high myopia using the excimer laser. Arch Ophthalmol 1993;3:1627-33. 